\title{
Suture anchor fixation strength with or without augmentation in osteopenic and severely osteoporotic bones in rotator cuff repair: a biomechanical study on polyurethane foam model
}

\author{
Mehmet Serhan $\mathrm{Er}^{1 *}$, Levent Altinel ${ }^{1}$, Mehmet Eroglu², Ozgur Verim³ ${ }^{3}$, Teyfik Demir ${ }^{4}$ and Halil Atmaca ${ }^{1}$
}

\begin{abstract}
Background: The purpose of the present study was to compare the results of various types of anchor applications with or without augmentation in both osteopenic and severely osteoporotic bone models.

Methods: Two different types of suture anchors were tested in severely osteoporotic (SOP) and osteopenic polyurethane (PU) foam blocks using an established protocol. An Instron machine applied static loading parallel to the axis of insertion until failure, and the mean anchor failure strengths were calculated. The mode of failure (anchor pullout, suture tear) was recorded. The anchors tested included the Corkscrew ${ }^{\circledast}$ (CS) (Arthrex Inc., Naples, FL, USA) (without augmentation, polymethylmethacrylate (PMMA)-augmented, and bioabsorbable tricalcium phosphate (TCP) cement-augmented) and Corkscrew ${ }^{\otimes}$ FT II (CS FT II) $5.5 \mathrm{~mm}$ (without augmentation as used routinely).

Results: The mean failure loads for both SOP and osteopenic PU foam blocks, respectively, were as follows: CS, 16.2 and $212.4 \mathrm{~N} ;$ CS with TCP, 75.2 and $396 \mathrm{~N} ;$ CS with PMMA, 101.2 and $528.8 \mathrm{~N} ;$ CS FT II, 13.8 and $339.8 \mathrm{~N}$.

Conclusions: Augmentation of CS with TCP or PMMA would be essential to SOP bones. In the osteopenic bone model, although anchor fixation augmented with PMMA is the best fixation method, CS augmented with TCP cement or CS FT II without any need for augmentation may also be used as an alternative.
\end{abstract}

Keywords: Suture anchor, Pullout, Osteoporosis, Rotator cuff repair, Tricalcium phosphate cement, Bone

\section{Background}

Rotator cuff tears (RCT) are common causes of shoulder pain and disability, especially in the elderly population with a high prevalence of osteopenia and osteoporosis. Management of these pathologies would require the use of many sophisticated methods (transposition of the intact subscapularis tendon to cover the superior defect, implantation of fascial autograft or allograft tissue, repair of the existing tendon more medially onto the articular surface, latissimus dorsi tendon transfer, free tendon transfer, or simple decompression with debridement of

\footnotetext{
* Correspondence: mserhan2005@hotmail.com

'Department of Orthopaedics and Traumatology, Faculty of Medicine,

Akdeniz University, Antalya 07985, Turkey

Full list of author information is available at the end of the article
}

the rotator cuff) [1]. The quality of cuff healing depends on multiple factors including the biomechanical properties of anchors, angle of insertion, anchor design, suture strength as well as the soft tissue, and bone quality. Because the local poor bone quality and systemic osteoporotic changes may compromise the success of RC repair in the elderly and result in suture anchor loosening, impaired tendon healing, and re-rupture of the RC [2], improvement in fixation quality of suture anchors becomes more essential to these patients.

Not only the systemic bone weakness but also the subchondral bone cysts in the proximal humerus due to chronic cuff tear can lead to poor bone quality and weaker fixation strength $[3,4]$. Before placing a suture anchor, the surgeon should decide which anchoring position will allow 
the most anatomic repair. If the suture anchor fails after it is placed, insertion of it to another site can be problematic because it will be a non-anatomic place and tension at the suture-tendon and anchor-bone interfaces will be increased. Increased tensions at those interfaces bring risks for failure of fixation. During the anchor fixation, those risks may be reduced by bone grafting [5], positioning the anchor subcortically [4], and augmentation of the fixation with polymethylmethacrylate (PMMA) or bioabsorbable tricalcium phosphate (TCP) cement $[3,6]$.

It was previously reported that augmentation of metal suture anchor fixation using bioabsorbable TCP cement and PMMA would increase the pullout strength of suture anchors from osteoporotic bones [3,6,7].

Previous studies about suture anchor strength with or without augmentation were conducted on a unique type of synthetic bone blocks. We aimed to compare the results of various anchor applications with or without augmentation in osteopenic and severely osteoporotic (SOP) bone models and to investigate if various types of suture anchors have different performances in bones with various densities. Do we really need to augment the fixation in osteopenic bones or do the fixation methods that are claimed to be more effective in osteopenic bones also work in SOP bones?

\section{Materials and methods}

\section{Experimental design overview}

In this study, two different types of suture anchors were tested in SOP and osteopenic polyurethane (PU) foam blocks. The study was conducted to compare the pullout strengths of the anchors and decide the strongest fixation tool and method.

\section{PU foam samples}

Two different PU foams that represent SOP and osteopenic bones were used [American Society for Testing and Materials (ASTM) grades 5 and 12, respectively] as testing mediums. The biomechanical properties of the bone models are given in Table 1. Synthetic bone materials are widely used in order to provide a more reliable test medium and uniformity between multiple testing groups unlike cadaveric samples. The ASTM F-1839 states that rigid polyurethane foam is an ideal material for the comparative testing of bone screws and other medical devices and instruments $[8,9]$.

Table 1 Biomechanical properties of severely osteoporotic and osteopenic bone models

\begin{tabular}{llcc}
\hline Density & Compressive strength & Compressive modulus \\
\hline (pcf) & $\mathbf{( g / c c )}$ & (MPa) & (MPa) \\
7.5 & 0.12 & 0.28 & 7.5 \\
20.2 & 0.324 & 8.4 & 20.2 \\
\hline
\end{tabular}

SOP samples (Figure 1) were supplied as blocks $(65 \mathrm{~mm} \times 50 \mathrm{~mm} \times 50 \mathrm{~mm})$ from Sawbones ${ }^{\bullet}$ Europe AB (Malmö, Sweden). Osteopenic blocks $(60 \mathrm{~mm} \times 60 \mathrm{~mm} \times$ $60 \mathrm{~mm}$ ) (Figure 2) were produced by the Department of Biomedical Engineering (TOBB University of Economics and Technology). Regular use of the latter foam blocks was approved previously [10].

\section{Suture anchors}

Two types of suture anchor (Figure 3) were investigated: Corkscrew $^{\circ}(5.5 \mathrm{~mm} \times 15 \mathrm{~mm})$ and Corkscrew ${ }^{\circ}$ FT II $(5.5 \mathrm{~mm} \times 16.3 \mathrm{~mm})$ (Arthrex, Naples, FL, USA).

Corkscrew $^{\circ}$ (CS) is not fully threaded with a $3-\mathrm{mm}$ part engaged in the applicator, and it has two strands of FiberWire $^{\circ}$ (Arthrex, Naples, FL, USA). Although Corkscrew ${ }^{\circ}$ FT II (CS FT II) was designed to maximize fixation in cortical bone, it was tested without any augmentation because in clinical practice, you cannot always manage to find enough cortical support especially in osteoporotic bones. This anchor is fully threaded and has thicker threads to increase the pullout strength when compared to CS.

\section{Anchor pullout testing}

Holes for anchor insertion with augmentation of TCP or PMMA were prepared perpendicular to the surface of the bone model via a Jamshidi needle (CareFusion, San Diego, CA, USA), as previously described, with a depth of $20 \mathrm{~mm}$ in all blocks [3].

CS was tested under three conditions: without augmentation, PMMA-augmented, and TCP-augmented. For those anchors augmented with TCP (Callos Inject, Acumed LLC, Hillsboro, OR, USA), 1.5 to $2.0 \mathrm{~mL}$ of material was injected by hand with the use of a syringe-like applicator device available with the TCP cement; a gentle backfill technique along the entire length of the pilot hole just before screw placement was performed. For those anchors applied with PMMA (Surgical Simplex ${ }^{\circ}$ P, Stryker International, Limerick, Ireland), the cement was prepared manually and inserted as soon as the cement was mixed until homogeneous, immediately placed into a 20-mL syringe while runny, and attached to a Jamshidi needle. PMMA was applied to the pilot holes by using the similar amount and backfill technique as performed with TCP. After application of TCP and PMMA, anchors were inserted to whole length. All procedures were conducted at $24^{\circ} \mathrm{C}$ and the humidity was $50 \%$.

Although in some of the previous studies $[2,6,11]$ sutures of the anchors were replaced with steel wires, we preferred to perform the tests in the original states of the anchors (two strands of \#2 FiberWire ${ }^{\circ}$ ). Because we do not use steel wires in clinical practice, we believe that it is more reasonable to assess the anchors without replacing wires to simulate what happens in vivo. Besides, the eyelet of CS FT II was made of fiber wire, and if we 

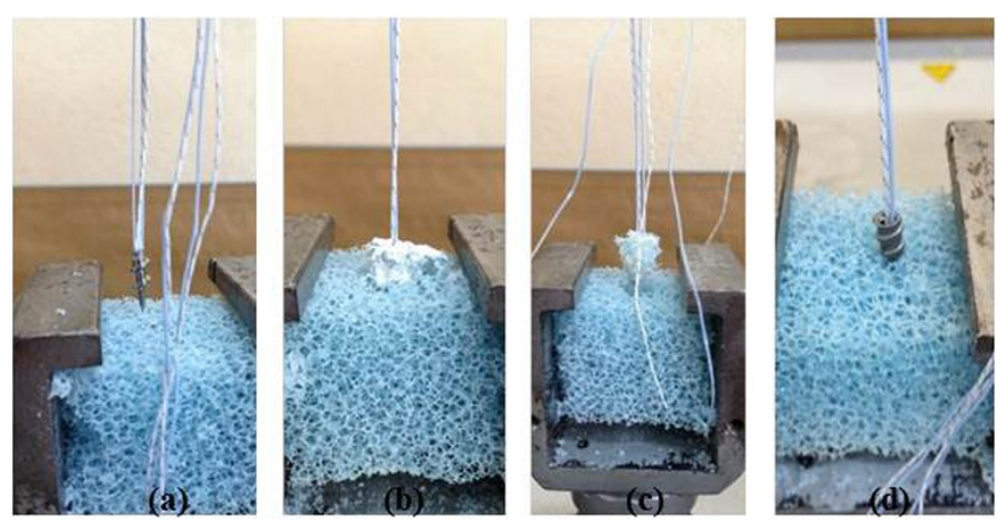

Figure 1 Tested specimens on severely osteoporotic polyurethane foam block. (a) Corkscrew ${ }^{\oplus}$. (b) TCP-augmented Corkscrew ${ }^{\oplus}$ (c) PMMA-augmented Corkscrew ${ }^{\oplus}$. (d) Corkscrew ${ }^{\oplus}$ FT II.

had replaced it with steel wires, the eyelet would break down against the wire.

Bone model blocks were secured to an Instron testing machine using clamping devices. One end of the suture was inserted in the screw's eyelet, and then this end was tied up with the other end of the suture on the metal bar, which was inserted in the upper fixture of the test setup (Figure 4). The length of the suture loop was $100 \mathrm{~mm}$ in all tests. The sutures were preloaded with $2 \mathrm{~N}$. To minimize the risk of suture tear, a cylindrical metal bar was used.

Instron 3300 testing machine (Instron Corp., Norwood, MA, USA) was used to pull out the anchors axially, and load versus displacement plots were recorded by its computer software. Axial tension was applied with $25 \mathrm{~mm} /$ min constant crosshead speed, and yield load was determined with 0.002 offset methods. For each group, five tests were performed. Failure criteria to stop the test were pulling out of the anchors with peak load from the PU foam blocks or tear of sutures.
All designed anchor systems were tested in accordance with ASTM F543 (ref.11 ASTM Standards, F543-07. Standard specification and test methods for metallic medical bone screws, 2002). Although the cyclic testing is closer to the clinical case, the pullout of the anchors is more prone to occur in the earlier postoperative period. So we applied static pullout tests in all four conditions.

\section{Statistical analysis}

Statistical analyses were performed by using SPSS 15.0 (SPSS Inc, Chicago, IL, USA). Differences in mechanical performance between different anchor constructs were analyzed by using analysis of variance (ANOVA). In order to understand which construct caused differences, data were analyzed by using post hoc Bonferroni. Statistical significance was defined as $p<0.05$.

\section{Results}

Pullout results of constructs are given in Table 2. It was seen that TCP augmentation increased the pullout strength
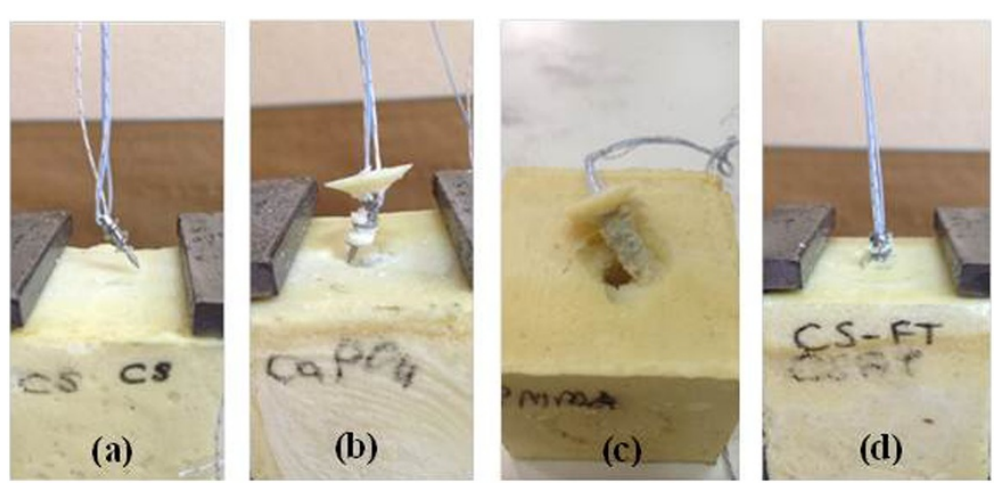

(b) TCP-augmented Corkscrew. (c) PMMA-augmented Corkscrew Figure 2 Tested sped
(d) Corkscrew ${ }^{\circledast} \mathrm{FT}$ 


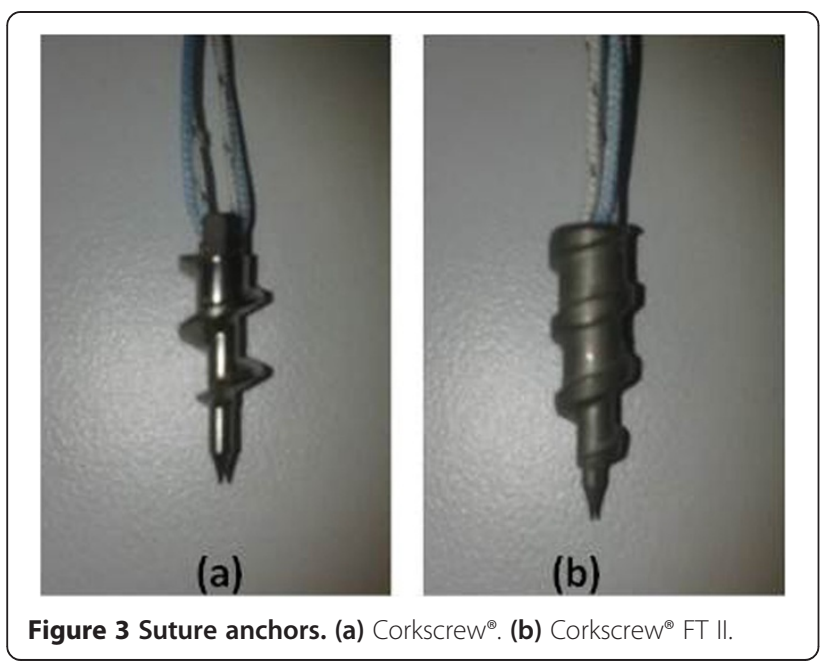

$364 \%$ on SOP samples and $86 \%$ on osteopenic samples when compared with CS with no cement. On the other hand, PMMA augmentation increased the pullout strength $524 \%$ on SOP blocks and $148 \%$ on osteopenic blocks when compared with the application of CS without cement. The pullout strength of PMMA augmentation was 34\% greater on SOP blocks and 33\% greater on osteopenic blocks when compared with TCP augmentation. These differences between CS + PMMA and CS + TCP were statistically insignificant in the SOP model while significant in the osteopenic bone model (Tables 3 and 4).

When the pullout strength of CS FT II values were compared with CS, it was seen that CS FT II exhibited 14\% lower pullout strength on SOP blocks and 59\% higher pullout strength on osteopenic blocks. CS FT II provided statistically significant augmentation in the osteopenic group ( $p=0.009)$ (Tables 3 and 4 ).

The severely osteoporotic PU foam block had a porous structure, so the failure always occurred in the boneanchor interface; on the other hand, on osteopenic PU foam blocks, mostly, the suture (two strands of \#2 FiberWire ${ }^{\circ}$ ) was torn. Tear of the suture showed that anchors can resist higher loads than the suture can.

\section{Discussion}

The quality of rotator cuff repair depends on the reliability of the mechanical strength of the construct and is determined by the weakest link in the chain. Compromised bone quality in the greater tuberosity (GT) due to systemic osteoporosis, immobilization after chronic RCT, subchondral bone cysts caused by chronic impingement, and prior surgery forms the weakest link in this chain $[4,6,11,12]$. The cancellous part of the GT rather than the cortical part is far more altered in these situations [4].

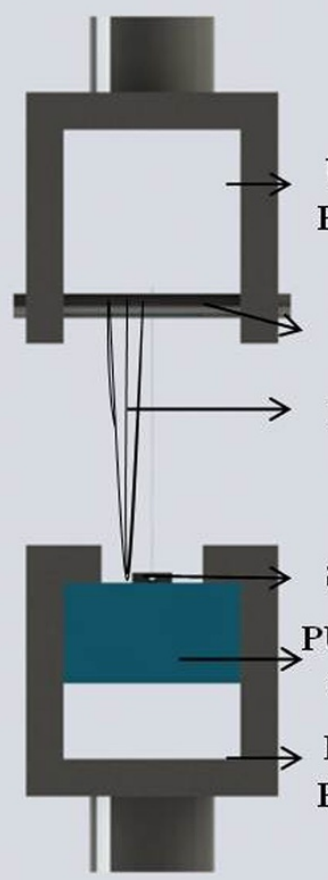

(a)

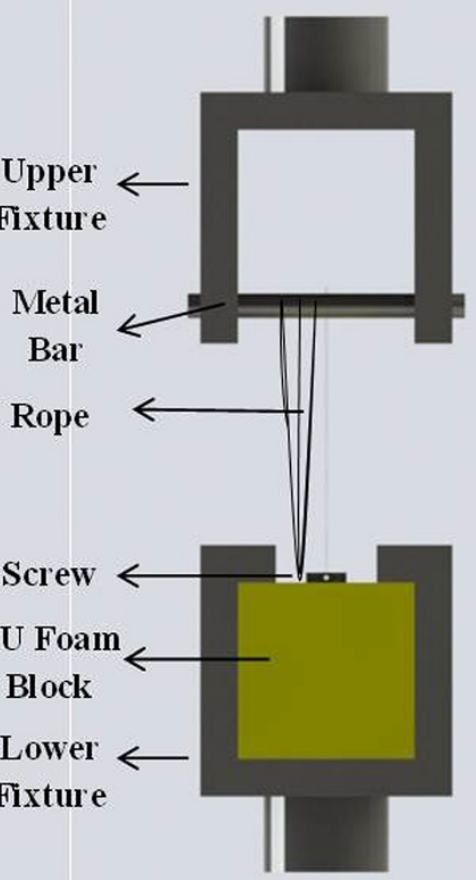

(b)

Figure 4 Test setup. (a) Severely osteoporotic. (b) Osteopenic. 
Table 2 Summary of results for both osteopenic and severely osteoporotic bone models

\begin{tabular}{|c|c|c|c|c|}
\hline & \multicolumn{2}{|c|}{ Severely osteoporotic } & \multicolumn{2}{|c|}{ Osteopenic } \\
\hline & Ultimate failure load (N) & Mode of failure & Ultimate failure load (N) & Mode of failure \\
\hline CS & $16.2 \pm 3.8$ & Foam & $212.4 \pm 64.2$ & Suture break \\
\hline CS + PMMA & $101.2 \pm 33.3$ & Foam & $528.8 \pm 45$ & Suture break \\
\hline CS FT II & $13.8 \pm 2.4$ & Foam & $339.8 \pm 48$ & Suture break \\
\hline $\mathrm{CS}+\mathrm{TCP}$ & $75.2 \pm 25.1$ & Foam & $396 \pm 52.7$ & Suture break \\
\hline
\end{tabular}

This is the first study to compare the effects of TCP and PMMA cements in the augmentation of suture anchor fixation on osteopenic and SOP bone models. While the best results for osteopenic bone models were provided by PMMA-augmented CS, both the TCP- and PMMA-augmented CS provided similar results on SOP bone models. These results show that PMMA has a superior effect in increasing pullout strength especially for osteopenic bones. Although CS FT II was designed to obtain higher pullout strength values, PMMA-augmented CS exhibited better test results.

We searched if the obtained values for pullout strength were high enough to resist failure in the early postoperative period or when a probable failure would occur. For massive tears involving the supraspinatus and infraspinatus tendons, the optimal postoperative shoulder immobilization postures are with the humerus elevated and externally rotated in changing angles according to the size of the defect in the rotator cuff, in order to minimize passive tension in the repaired tendon(s) during the immediate postoperative period and improve the potential for healing [13]. The test results, with the exception of CS and CS FT II pullout tests on SOP bone models, are above the tensile forces of these tendons with the postoperative shoulder immobilization postures [13] (Figure 5).

Barber et al. [14] concluded in their study that there is no correlation with the pullout strength of the screw type anchor and bone mineral density (BMD); however, other studies trying to characterize the bone architecture of the GT and investigating the correlation between pullout strength and BMD in different locations suggested that the bone density varies between individuals but is highest at the posteromedial aspect of the GT $[4,12]$ and the pullout strength of anchor fixations increases with BMD $[2,11]$.

Researchers have investigated whether this emerged problem can be solved by using PMMA or TCP cement

Table 3 Statistical results of anchor fixations on the severely osteoporotic bone model

\begin{tabular}{lccc}
\hline & CS & CS + PMMA & CS FT II \\
\hline CS + PMMA & 0.000 & & \\
CS FT II & 1.000 & 0.000 & \\
CS + TCP & 0.002 & 0.407 & 0.002 \\
\hline
\end{tabular}

augmentation techniques as previously used in fracture fixation and spine surgery [15-18]. In their cadaveric study in which the specimens were not osteoporotic, Oshtory et al. [3] reported an increase of $29 \%$ in the pullout strength of suture anchor fixation augmented with TCP cement. On the other hand, Giori et al. stated that augmentation of suture anchor fixation with PMMA bone cement whether the suture anchor hole is stripped results in more cycles to failure and greater maximum load compared with non-PMMA-augmented suture anchors in intact bone. They found that the average maximum load carried by the suture anchor was $71 \%$ greater for anchors placed in PMMA-augmented holes compared with anchors inserted into intact bone (294 versus 172 N) [6]. Collinge et al. [15] compared TCP and PMMA in augmentation models using $4.5-\mathrm{mm}$ cortical screws inserted into synthetic osteoporotic bone models. They reported that both cements perform mechanically similar as a screw augmentation material.

PU foam bone models, which are homogeneous, simulate the biomechanical properties of native bone [19], and unlike stripped or drilled holes in cadaveric bones, they eliminate the possibility of encountering a healthy trabecular structure around the anchor that PMMA would interdigitate. The average pullout forces that we report in this study for the SOP model are smaller than those reported by both Meyer et al. and Giori et al., probably because both stripped holes (the holes created by the pullout of a suture anchor) [6] and drilled holes in another study [4] had healthy cancellous bone surrounding these holes so that PMMA would interdigitate. We also used original sutures of anchors instead of steel wires and so preferred to test their original status not to eliminate tear of the suture as a mode of failure.

Although the average pullout forces of PMMAaugmented anchors are greater than those of TCPaugmented ones, injection of PMMA into a suture

Table 4 Statistical results of anchor fixations on the osteopenic bone model

\begin{tabular}{lccc}
\hline & CS & CS + PMMA & CS FT II \\
\hline CS + PMMA & 0.000 & & \\
CS FT II & 0.009 & 0.000 & \\
CS + TCP & 0.000 & 0.007 & 0.678 \\
\hline
\end{tabular}




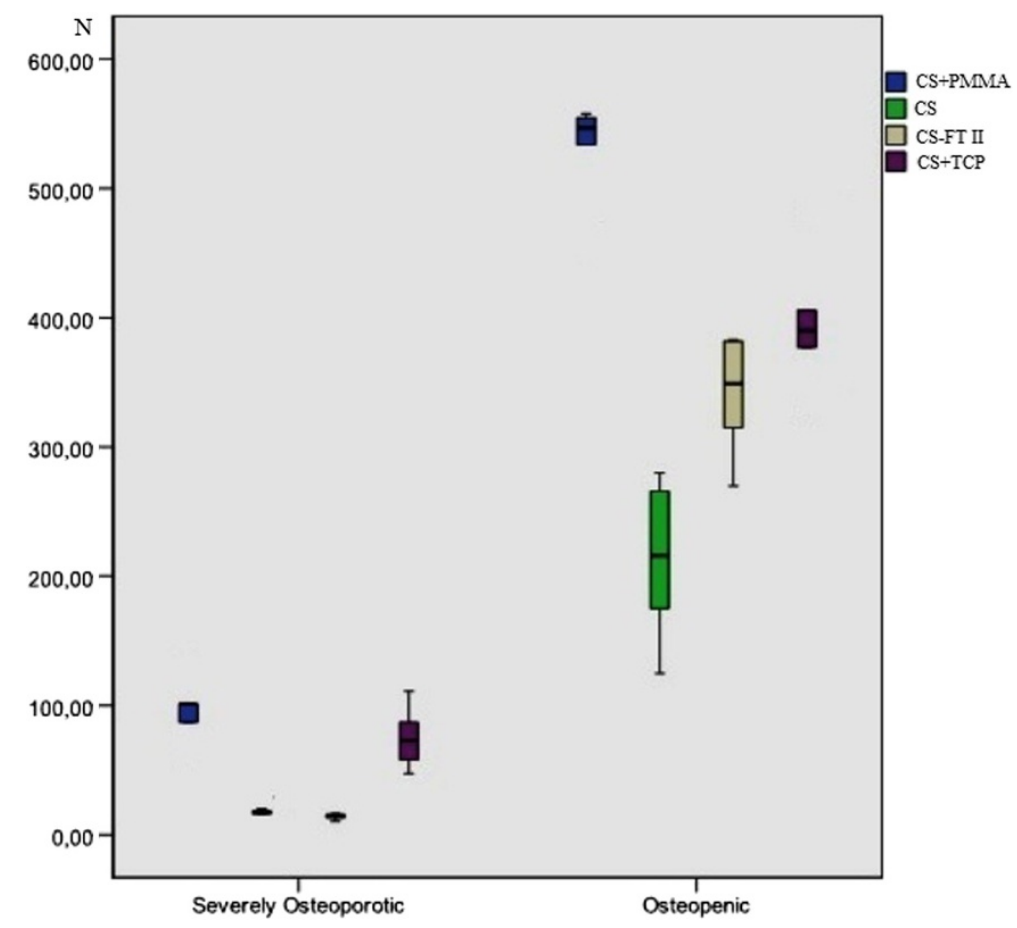

Figure 5 Box plot revealing the results of the pullout tests on the severely osteoporotic and osteopenic bone models.

anchor hole without spilling out of the hole is technically demanding when performing arthroscopically and it should be done through a mini-open approach to avoid the spilling out of PMMA particles in the joint space [6]. The cement would not be so runny to avoid leakage from the hole injected in. Otherwise, the cement particles may act as foreign bodies in the joint space [6]. Within this scope of view, augmentation with TCP cement has an advantage as application of it by a Jamshidi needle could easily be adapted for arthroscopy. Penetration of the cement into the intertrabecular space reaches approximately 0.1 to $2 \mathrm{~mm}$ in a normal cancellous bone, but in a SOP bone, further and perhaps unexpected penetration might occur [7], so cemented anchor failure may cause larger defects than non-cemented anchor failure.

Although the cement reaches $90 \%$ of its strength after $10 \mathrm{~min}$, it was allowed to cure for $24 \mathrm{~h}$ before mechanical testing. This waiting period would not be applicable in clinical practice, because the construct would likely be loaded after surgery before the curing period was completed. It is not clear whether restriction of load bearing by a shoulder immobilizer would eliminate this problem or not. This restriction may reduce the load on the osteopenic construct to subfailure levels but not on the SOP construct.

The static single pullout to failure technique seems as a limitation. But as we focused on the early postoperative failure which would be simulated by static pullout testing, we did not perform a cyclic loading test. Then, we placed the anchor sutures with the angle of $90^{\circ}$ to the surface of bone models, not with the angle of $45^{\circ}$ to $90^{\circ}$ (the so-called dead man's angle) [20], and we used linear pullout axis in line with the anchor's insertion that does not replicate the physiologic pull of the supraspinatus tendon [21]. We thought that this represented a worst-case scenario and would be more suitable to test the anchoring capacity of the suture anchor. Moreover, when an anchor is tested by pulling out axially, it can be thought that failure would occur at higher values in physiological conditions than the measured ones. However, RC repairs made with the screw-in suture anchor inserted at $90^{\circ}$ to the superior junction of the greater tuberosity and the humeral head articular surface provided better soft tissue fixation stability than repairs made with the anchors inserted at the dead man's angle of $45^{\circ}$ [20]. We used a fully cancellous bone model to simulate the worst condition; however, this would decrease the pullout strength of the fully threaded anchors (such as CS FT II) which mainly anchor the cortical bone. Further studies may explain how it differs to test such anchors in a fully cancellous and a corticocancellous bone model.

\section{Conclusion}

This study showed that in patients with SOP bones, fixation of the ruptured RC with any of these anchors used in this study without augmentation is not clinically applicable as it will most probably fail. Therefore, augmentation with TCP or PMMA would be essential to those 
patients. For patients with osteopenic bones, although CS augmented with PMMA performed best, CS FT II or CS augmented with TCP may also be used as an alternative choice of fixation for $\mathrm{RC}$ repairs. If possible, the bone mineral density may be determined preoperatively to decide if and how augmentation should be performed.

\section{Competing interests}

The authors declare that they have no competing interests.

\section{Authors' contributions}

MSE, LA, and ME participated in the study design, carried out the literature search, performed the data interpretation, and drafted the manuscript. OV and TD performed the biomechanical tests. HA performed the statistical analysis and was involved in revising the manuscript. All authors read and approved the final manuscript.

\section{Disclosure}

The authors or members of their family have not received any financial remuneration related to the subject of the article.

\section{Author details}

${ }^{1}$ Department of Orthopaedics and Traumatology, Faculty of Medicine Akdeniz University, Antalya 07985, Turkey. ${ }^{2}$ Department of Orthopaedics and Traumatology, Faculty of Medicine, Afyon Kocatepe University, Afyonkarahisar 03200, Turkey. ${ }^{3}$ Department of Mechanical Engineering, Faculty of Technology, Afyon Kocatepe University, Afyonkarahisar 03200, Turkey. ${ }^{4}$ Department of Biomedical Engineering, TOBB University of Economics and Technology, Ankara 06520, Turkey.

Received: 26 December 2013 Accepted: 9 June 2014

Published: 22 August 2014

\section{References}

1. Jones CK, Savoie FH 3rd: Arthroscopic repair of large and massive rotator cuff tears. Arthroscopy 2003, 19:564-571.

2. Tingart MJ, Apreleva M, Lehtinen J, Zurakowski D, Warner JJ: Anchor design and bone mineral density affect the pull-out strength of suture anchors in rotator cuff repair: which anchors are best to use in patients with low bone quality? Am J Sports Med 2004, 32:1466-1473.

3. Oshtory R, Lindsey DP, Giori NJ, Mirza FM: Bioabsorbable tricalcium phosphate bone cement strengthens fixation of suture anchors. Clin Orthop Relat Res 2010, 468:3406-3412

4. Meyer DC, Fucentese SF, Koller B, Gerber C: Association of osteopenia of the humeral head with full-thickness rotator cuff tears. J Shoulder Elbow Surg 2004, 13:333-337.

5. Kim KC, Rhee KJ, Shin HD, Kim YM: Arthroscopic footprint reconstruction of a bone cyst-associated rotator cuff tear. Knee Surg Sports Traumatol Arthrosc 2007, 15:1486-1488

6. Giori NJ, Sohn DH, Mirza FM, Lindsey DP, Lee AT: Bone cement improves suture anchor fixation. Clin Orthop Relat Res 2006, 451:236-241.

7. Meyer DC, Jacob HA, Pistoia W, von Roll A, Gerber C: The use of acrylic bone cement for suture anchoring. Clin Orthop Relat Res 2003, 410:295-302.

8. Ricci WM, Tornetta P 3rd, Petteys T, Gerlach D, Cartner J, Walker Z, Russell TA: A comparison of screw insertion torque and pullout strength. J Orthop Trauma 2010, 24:374-378.

9. Pfeiffer FM, Abernathie DL, Smith DE: A comparison of pullout strength for pedicle screws of different designs: a study using tapped and untapped pilot holes. Spine 2006, 31:E867-870.

10. Arslan AK, Demir T, Ormeci MF, Camuscu N, Tureyen K: Postfusion pullout strength comparison of a novel pedicle screw with classical pedicle screws on synthetic foams. Proc Inst Mech Eng H 2013, 227:114-119.

11. Meyer DC, Mayer J, Weber U, Mueller A, Koch PP, Gerber C: Ultrasonically implanted PLA suture anchors are stable in osteopenic bone. Clin Orthop Relat Res 2006, 442:143-148.

12. Kirchhoff $C$, Braunstein $V$, Milz S, Sprecher CM, Fischer F, Tami A, Ahrens $P$, Imhoff $A B$, Hinterwimmer $S$ : Assessment of bone quality within the tuberosities of the osteoporotic humeral head: relevance for anchor positioning in rotator cuff repair. Am J Sports Med 2010, 38:564-569.
13. Jackson M, Sylvestre E, Bleau J, Allard P, Begon M: Estimating optimal shoulder immobilization postures following surgical repair of massive rotator cuff tears. J Biomech 2013, 46:179-182.

14. Barber FA, Feder SM, Burkhart SS, Ahrens J: The relationship of suture anchor failure and bone density to proximal humerus location: a cadaveric study. Arthroscopy 1997, 13:340-345.

15. Collinge C, Merk B, Lautenschlager EP: Mechanical evaluation of fracture fixation augmented with tricalcium phosphate bone cement in a porous osteoporotic cancellous bone model. J Orthop Trauma 2007, 21:124-128.

16. Elder S, Frankenburg E, Goulet J, Yetkinler D, Poser R, Goldstein S: Biomechanical evaluation of calcium phosphate cement-augmented fixation of unstable intertrochanteric fractures. J Orthop Trauma 2000, 14:386-393.

17. Moore DC, Frankenburg EP, Goulet JA, Goldstein SA: Hip screw augmentation with an in situ-setting calcium phosphate cement: an in vitro biomechanical analysis. J Orthop Trauma 1997, 11:577-583.

18. Moore DC, Maitra RS, Farjo LA, Graziano GP, Goldstein SA: Restoration of pedicle screw fixation with an in situ setting calcium phosphate cement. Spine 1997, 22:1696-1705.

19. Gibson LJ, Ashby MF: Cancellous bone. In Cellular Solids. New York: Pergamon; 1988:316-331.

20. Strauss E, Frank D, Kubiak E, Kummer F, Rokito A: The effect of the angle of suture anchor insertion on fixation failure at the tendon-suture interface after rotator cuff repair: deadman's angle revisited. Arthroscopy 2009, 25:597-602

21. Schneeberger AG, von Roll A, Kalberer F, Jacob HA, Gerber C: Mechanical strength of arthroscopic rotator cuff repair techniques: an in vitro study. J Bone Joint Surg Am 2002, 84-A:2152-2160.

doi:10.1186/1749-799X-9-48

Cite this article as: Er et al:: Suture anchor fixation strength with or without augmentation in osteopenic and severely osteoporotic bones in rotator cuff repair: a biomechanical study on polyurethane foam model. Journal of Orthopaedic Surgery and Research 2014 9:48.

\section{Submit your next manuscript to BioMed Central and take full advantage of:}

- Convenient online submission

- Thorough peer review

- No space constraints or color figure charges

- Immediate publication on acceptance

- Inclusion in PubMed, CAS, Scopus and Google Scholar

- Research which is freely available for redistribution 